\title{
Superior Mesenteric Artery syndrome following Augmentation Cystoplasty
}

Amr K. Salama, MD, Khalil N. Saad, MD, Mark P. Cain, MD

Department of Urology, Indiana University School of Medicine

\section{Corresponding Author:}

Amr K. Salama, MD

${ }^{a} 705$ Riley Hospital Drive, Riley Outpatient Center, 4230, Indianapolis, IN 46202

Phone: 3172782476; e-mail: aksalama@iu.edu

Saad:Knsaad@iu.edu ; Cain: Mpcain@iupui.edu

Abstract: 36 Manuscript word count: 980

Keywords: Pediatrics; superior mesenteric artery syndrome; bladder augmentation

\section{Acknowledgements: None}

\section{Disclosures: None}

This is the author's manuscript of the article published in final edited form as:

Salama, A. K., Saad, K. N., \& Cain, M. P. (2019). Superior Mesenteric Artery syndrome following Augmentation Cystoplasty. Urology. https://doi.org/10.1016/j.urology.2019.06.013 
Abstract:

Superior Mesenteric Artery Syndrome (SMAS) is a rare condition of external duodenal compression in the angle between the superior mesenteric artery and aorta. We report a case of SMAS following augmentation cystoplasty in a young patient.

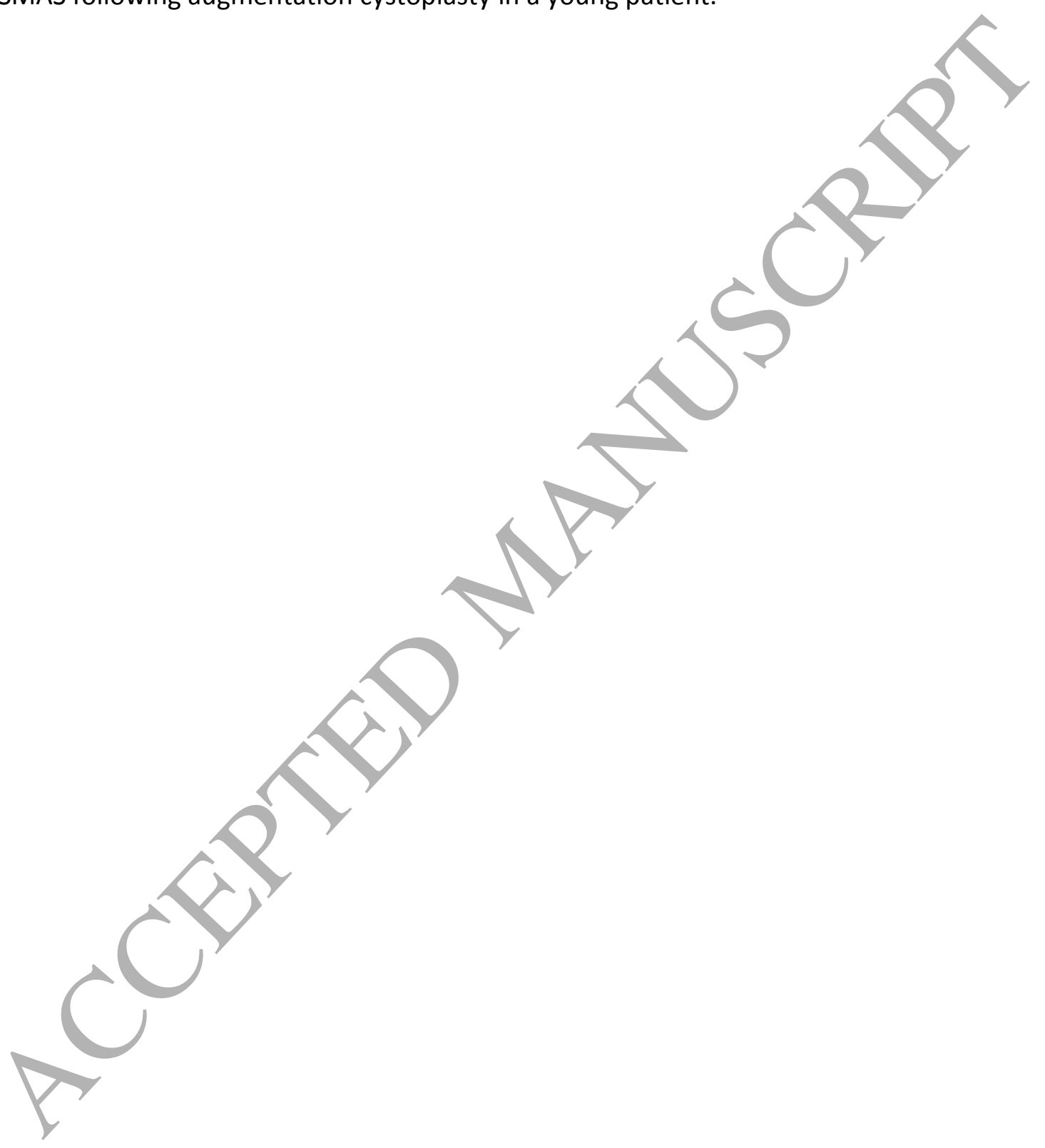




\section{Introduction:}

Superior mesenteric artery Syndrome (SMAS) is a rare condition that usually presents with symptoms of upper gastrointestinal (GI) obstruction due to extrinsic compression of the third part of duodenum between the abdominal aorta posteriorly and superior mesenteric artery (SMA) anteriorly. Several predisposing factors have been described, however; severe weight loss is considered the most significant. Reduction in the angle and distance between the aorta and the superior mesenteric artery causes compression of the duodenum. Conservative treatment plays a major role in such cases; however, failure of such measures may warrant surgical intervention.

\section{The Case:}

An 8-year-old male, who was born with bilateral high-grade vesicoureteral reflux (VUR) developed non-neurogenic - neurogenic bladder with a small bladder capacity complicated by persistent high grade left VUR that led to marked deterioration in left renal function to $20 \%$. Due to poor compliance and difficulty with intermittent catheterization, a cutaneous vesicostomy was performed at age 2 years. Spinal MRI excluded possible spinal cord lesions. At age 6 the vesicostomy was closed and the left ureter reimplanted with intradetrusor OnabotulinumA (ВОТОX) injection.

Because of persistent poor bladder compliance, an augmentation cystoplasty with appendicovesicostomy is planned. Intraoperatively the mesoappendix found to be inflamed, therefore appendectomy was performed, and a Monti channel was created along with the planned ileocystoplasty. The ileal segment had mucosal inflammation of the bowel and chronic mesenteric adenopathy with chronic inflammation on biopsy. 
The patient had an unremarkable postoperative course and was discharged after 5 days. He presented to the emergency department after two weeks with a complaint of lowgrade fever, vomiting and abdominal pain. Clinical examination at that time was normal except for documented weight loss (Figure 1). Urine culture was positive and treated with intravenous antibiotics; however, other lab results including work up for inflammatory bowel disease returned normal. He had prolonged delay in return of bowel function with bilious vomiting. Upper gastrointestinal series were obtained (Figure 2) as requested by gastrointestinal consultation; it demonstrated dilatation of the first and second part of duodenum, delayed contrast passage from the second portion into the third portion of the duodenum, and no distal small bowel obstruction. This confirmed the diagnosis of (SMAS) based on clinical and imaging findings. Nasojejunostomy tube was placed to start enteral feeding, which he tolerated well. After a three-month course of enteral tube feeding, he eventually gained significant weight, improvement was noted as the patient started to tolerate oral diet and was confirmed with upper GI series.

The patient was readmitted later to the hospital on two separate occasions (several months apart) with the complaint of persistent vomiting and inability to tolerate feeding. Significant weight loss of what was previously gained was documented in the two occasions. Upon starting gradual NJ feeding the patient started to show slight improvement. He was discharged four weeks later on close follow up of his weight gain, feeding tolerance as he gradually increases the amount of diet intake. Currently the patient is showing improvement in his condition as his weight started to rise to the lower margin of normal percentile for his age. 


\section{Discussion}

Intestinal segments in various forms have been used to reconstruct the urinary tract since 1888. (1) Urinary diversion was the most common method in children ${ }^{(2)}$ until augmentation cystoplasty was applicable when Lapides et al ${ }^{(3)}$ showed the effectiveness of clean intermittent catheterization (CIC) to empty the native or augmented bladder. However, inclusion of bowel segments in urinary bladder reconstruction is associated with different metabolic consequences and late surgical complications depending on the part of GI tract used. (4) In our case, ileum was used in augmentation and Monti channel, however; the patient presented with upper GI obstructive symptoms, this made the diagnosis more challenging.

SMAS, also known as Willkie's syndrome, or cast syndrome is a rare disorder characterized by external compression of the third part of the duodenum between the superior mesenteric artery anteriorly and aorta posteriorly which leads to upper gastrointestinal obstruction. $^{(5,6)}$

Rokitansky was the first to describe it in $1842^{(7)}$ during an autopsy, followed by a more detailed description reported by Willet in 1878 with a series of 64 patients ${ }^{(8)}$. It has a reported incidence of $0.013 \%-0.3 \% .^{(9,10)}$ The largest case series of SMA syndrome in children was reported by Biank et al ${ }^{(8)}$ where 22 cases were diagnosed over a period of 20 years.

Burrington described four broad etiological categories; congenital due to high insertion of the ligament of Treitz, rapid weight loss, or rapid growth without weight gain especially in young patients and hyperextension of the spine in cast or brace. ${ }^{(7)}$ The associated loss of 
retroperitoneal fat causes the acute angulation of superior mesenteric artery which is the defining feature of SMA syndrome. ${ }^{(11)}$

The severity of symptoms depends on the degree of compression of the duodenum as defined by the aorto-mesenteric angle. ${ }^{(12)}$ Patients may present either with chronic insidious symptoms (e.g. long standing vague abdominal pain associated with vomiting) or with acute exacerbation of chronic symptoms (e.g. signs and symptoms of duodenal obstruction). Less commonly patients present with early satiety with sensation of fullness due to increased gastric distention and increased transit time. ${ }^{(13)}$

Diagnosis of SMAS is quite challenging because of the vague insidious onset. Clinical suspicion should be raised in the context of a patient with upper Gl obstructive symptoms and history of severe weight loss. Different diagnostic methods can be used, including barium radiography which could demonstrate dilation of first and second part of duodenum associated with anti-peristaltic flow of barium proximally and 4-6 hours delay in gastrojujenal transit time. ${ }^{(14)}$ Contrast enhanced computed tomography or magnetic resonance angiography allows visualization of the vascular/compression and measuring the aorto- mesenteric angle. Endoscopy can be helpful in some cases, as it can visualize a pulsatile extrinsic compression suggesting the SMA syndrome.

Conservative measures have been the traditional treatment for SMA syndrome; this includes gastric decompression and bypass using a NJ tube, clear liquid diet or parenteral nutrition aiming at increasing retroperitoneal fat deposition. ${ }^{(14)}$ In cases of failed conservative measures, surgery can be offered in the form of duodenojejunostomy with success rate up to 
$90 \% .{ }^{(13)}$ A less invasive surgical procedure is to lyse the ligament of Treitz with mobilization of the duodenum, however a failure rate of $25 \%$ is reported. ${ }^{(15)}$

\section{Conclusion:}

To our knowledge, this is the first case of SMAS following urologic reconstructive surgery in a child. We are reporting this case to highlight the SMAS as very rare but possible cause for post augmentation or urologic reconstruction persistent GI symptoms.

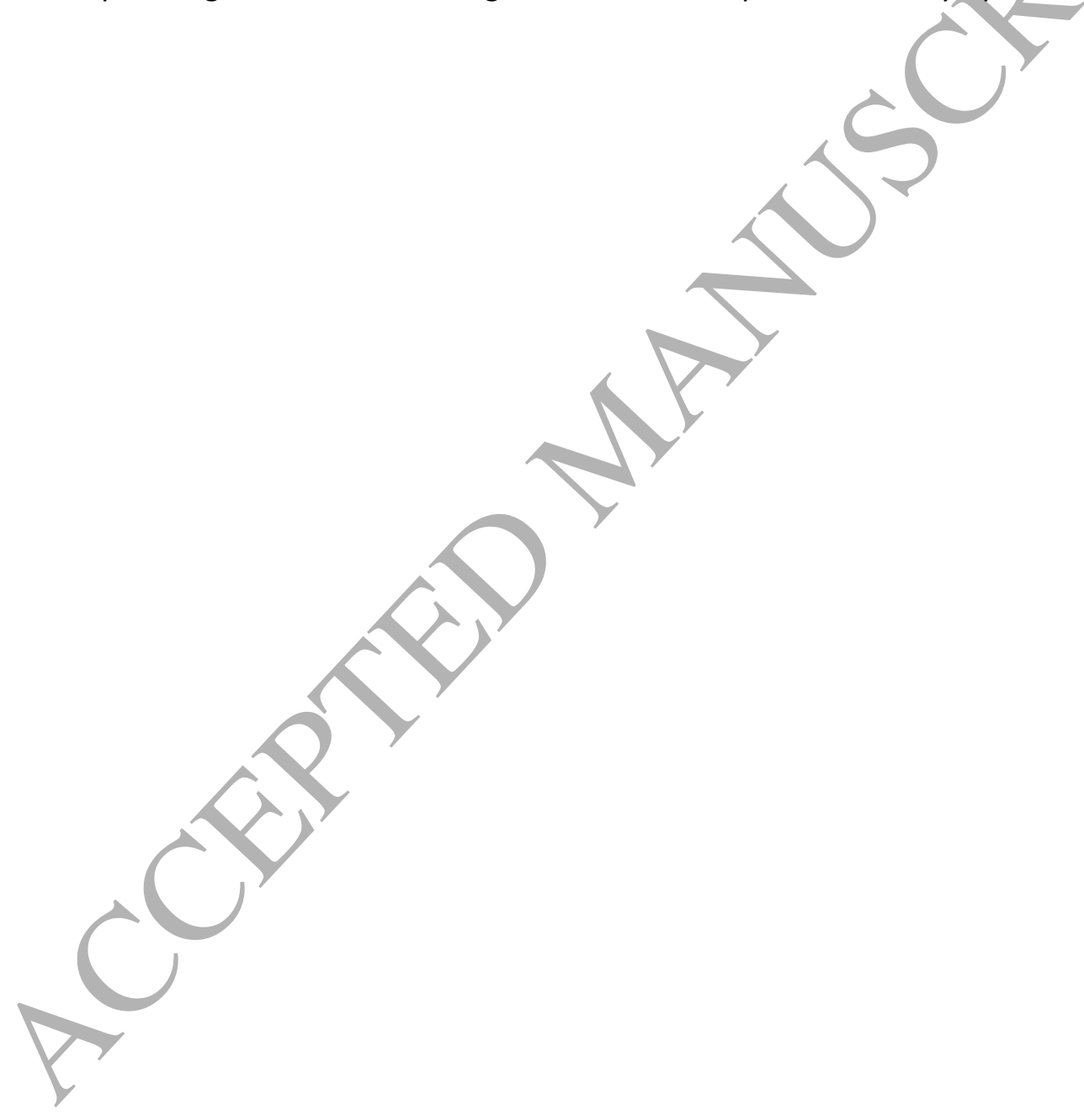




\section{References:}

1. Thuroff JW, Alken P, Riedmiller H, Jacobi GH, Hohenfellner R. The Mainz pouch for augmentation or substitution of the bladder and continent diversion. Semin Urol. 1987;5(1):69-79.

2. Shapiro SR, Lebowitz R, Colodny AH. Fate of 90 children with ileal conduit urinary diversion a decade later: analysis of complications, pyelography, renal function and bacteriology. J Urol. $1975 ; 114(2): 289-95$.

3. Lapides J, Diokno AC, Silber SJ, Lowe BS. Clean, intermittent self-catheterization in the treatment of urinary tract disease. Trans Am Assoc Genitourin Surg. 1971;63:92-6.

4. Mitchell ME. Bladder augmentation in children: where have we been and where are we going? BJU Int. 2003;92 Suppl 1:29-34.

5. Zaraket V, Deeb L. Wilkie's Syndrome or Superior Mesenteric Artery Syndrome: Fact or Fantasy? Case Rep Gastroenterol. 2015;9(2):194-9.

6. Ugras M, Bicer S, Coskun FT, Romano E, Ekci B. Superior mesenteric artery syndrome: A rare but life threatening disease. Turk J Emerg Med. 2017;17(2):70-2.

7. Burrington JD. Superior mesenteric artery syndrome in children. Am J Dis Child. 1976;130(12):1367-70.

8. Biank V, Werlin S. Superior mesenteric artery syndrome in children: a 20-year experience. J Pediatr Gastroenterol Nutr. 2006;42(5):522-5.

9. Anderson JR, Earnshaw PM, Fraser GM. Extrinsic compression of the third part of the duodenum. Clin Radiol. 1982;33(1):75-81.

10. Lee CS, Mangla JC. Superior mesenteric artery compression syndrome. Am J Gastroenterol. $1978 ; 70(2): 141-50$.

11. Tsirikos Al, Jeans LA. Superior mesenteric artery syndrome in children and adolescents with spine deformities undergoing corrective surgery. J Spinal Disord Tech. 2005;18(3):263-71.

12. Baltazar U, Dunn J, Floresguerra C, Schmidt L, Browder W. Superior mesenteric artery syndrome: An uncommon cause of intestinal obstruction. Southern Med J. 2000;93(6):606-8.

13. Mandarry MT, Zhao L, Zhang C, Wei ZQ. A comprehensive review of superior mesenteric artery syndrome. Eur Surg. 2010;42(5);229-36.

14. Welsch T, Buchler MW, Kienle P. Recalling superior mesenteric artery syndrome. Digest Surg. 2007;24(3):149-56.

15. Merrett ND, Wilson RB, Cosman P, Biankin AV. Superior mesenteric artery syndrome: diagnosis and treatment strategies. J Gastrointest Surg. 2009;13(2):287-92. 


\section{Figure Legends:}

Figure 1: A graph showing documented weight changes and its relation to uro-surgery, SMAS, and follow up visits.

Weight

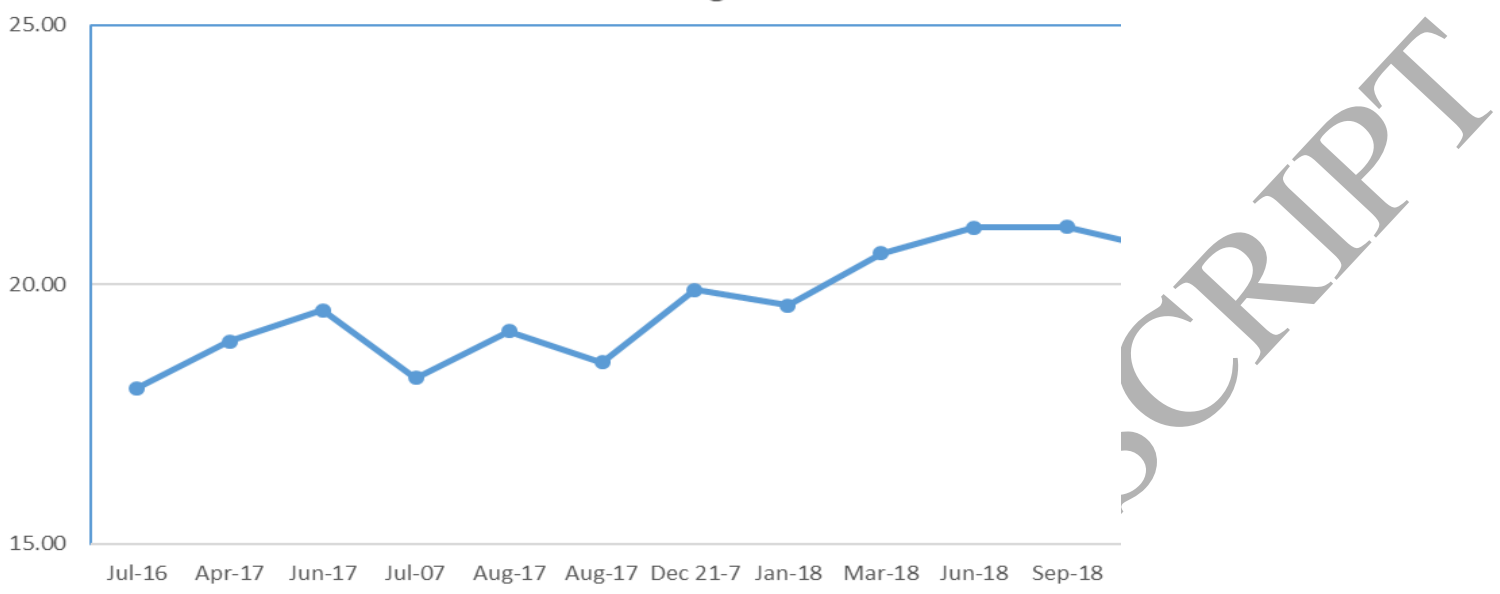

Figure 2: UGI series demonstrating dilatation of proximal duodenum and delayed passage of contrast through the distal part of duodenum

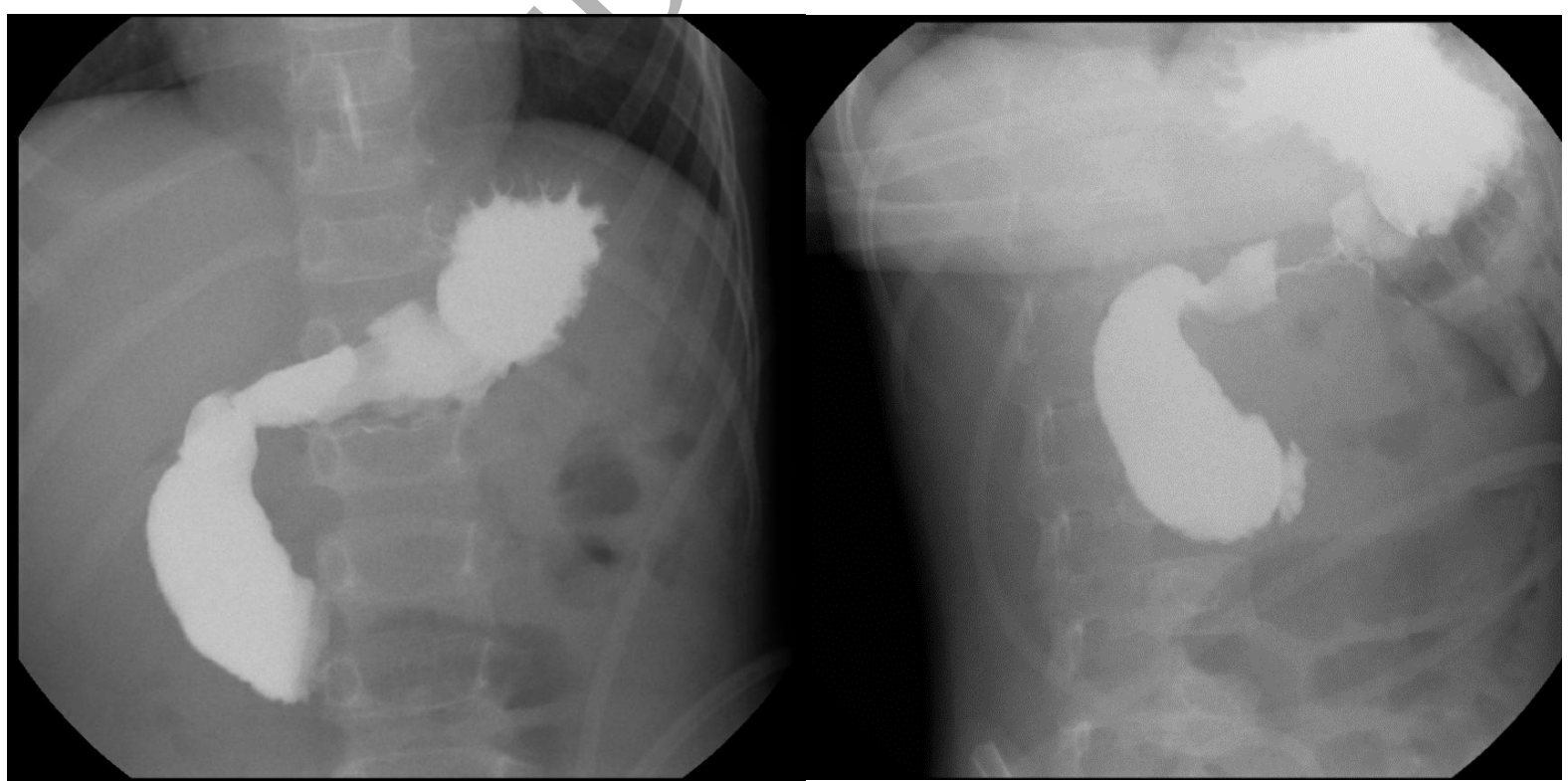

